\title{
Internal Inertia-Gravity Waves in the Tropical Lower Stratosphere Observed by the Arecibo Radar
}

\author{
YASUYUKI MAEKaWA \\ Radio Atmospheric Science Center, Kyoto University, Uji 611, Japan \\ SHOICHIRO FUKAO \\ Department of Electrical Engineering, Kyoto University, Kyoto 606, Japan
}

TORU SATO

Shigaraki MU Observatory, Radio Atmospheric Science Center, Kyoto University, Shigaraki, Shiga, 529-18, Japan

SUSUMU Kato

Radio Atmospheric Science Center, Kyoto University, Uji 611, Japan

RONALD F. WOODMAN

Instituto Geofisico del Perú, Sector Educación, Lima 100, Perú

(Manuscript received 9 September 1983, in final form 22 June 1984)

\section{ABSTRACT}

\begin{abstract}
High-resolution upper tropospheric and lower stratospheric $(5-30 \mathrm{~km})$ wind data were obtained during three periods from 1979 to 1981 with the aid of the high-power UHF radar at Arecibo, Puerto Rico $\left(18.4^{\circ} \mathrm{N}\right.$, $66.8^{\circ} \mathrm{W}$ ). A quasi-periodic wind oscillation with an apparent period of 20-50 h was observed between 16 and $20 \mathrm{~km}$ in every experiment. The amplitude of both zonal and meridional wind components was $\sim 2 \mathrm{~m}$ $\mathrm{s}^{-1}$, and the vertical wavelength $\sim 2 \mathrm{~km}$. The direction of the wind associated with this oscillation rotated clockwise with time, as seen for inertia-gravity waves in the Northern Hemisphere.

The wave disappeared near $20 \mathrm{~km}$ where the mean zonal flow had easterly shear with height. This phenomenon is discussed in terms of wave absorption at a critical level. It is suggested that the wave had a westward horizontal phase speed of $10-20 \mathrm{~m} \mathrm{~s}^{-1}$. The intrinsic period and the horizontal wavelength at the wave-generated height are inferred to be $20-30 \mathrm{~h}$ and $\sim 2000 \mathrm{~km}$, respectively, from the relationship based on $f$-plane theory that the Doppler-shifted wave frequency approaches the Coriolis frequency at the critical level. The vertical group velocity estimated from the dispersion equation on the $f$-plane closely agrees with the ascending rate of the observed wave packets at each height.

In addition, each observation showed the presence of another type of oscillation with somewhat longer vertical wavelength in the lower stratosphere. If we assume the same intrinsic period and horizontal scale for this oscillation as for the abovementioned smaller vertical-scale wave at the tropopause level, the observed period and vertical structure are well described in terms of an internal inertia-gravity wave propagating to the opposite side in the horizontal plane.
\end{abstract}

\section{Introduction}

In the equatorial lower stratosphere, the vertically propagating internal wave disturbances resolved first by observational studies were mixed Rossby-gravity waves (Yanai and Maruyama, 1966) and Kelvin waves (Wallace and Kousky, 1968). These proved to be planetary-scale waves described in terms of the tidal equation on the equatorial beta-plane (Lindzen, 1967; Lindzen and Matsuno, 1968; Holton and Lindzen, 1968). In addition, some fragmentary observations from an extensive balloon launching experiment showed the existence of mesoscale wave perturbations which were revealed by multilayered structures of wind profiles (e.g., Madden and Zipser, 1970). Recently, Cadet and Teitelbaum (1979) investigated such quasi-periodic oscillations in relation to internal inertia-gravity waves by using data from successively released balloons over the eastern equatorial Atlantic Ocean in the northern summer of 1974 (during GATE). These waves are characterized by a very short vertical wavelength of $1-2 \mathrm{~km}$ and a wave period of $\sim 35 \mathrm{~h}$ (ground bacod). The horizontal phase speed relative to the ground is inferred to be

(c) 1984 American Meteorological Society 
about $-20 \mathrm{~m} \mathrm{~s}^{-1}$ (westward), giving a horizontal wavelength of $\sim 2400 \mathrm{~km}$.

Recent development of the high-power VHF/UHF radar technique has made it possible to observe the lower stratosphere with fine time and height resolutions (e.g., Balsley and Gage, 1980; Röttger, 1980; Woodman, 1980). In our previous study (Sato and Woodman, 1982a), we described similar small verticalscale structures of the wind profiles observed by the Arecibo, Puerto Rico $\left(18.4^{\circ} \mathrm{N}, 66.8^{\circ} \mathrm{W}\right) 430 \mathrm{MHz}$ radar with $150 \mathrm{~m}$ height resolution in October 1979. Here, detailed discussions of such wind fluctuations, including June 1979 and March 1981 observational data, are made in light of internal inertia-gravity waves.

We briefly summarize the present experimental technique in Section 2, since the detailed descriptions have been presented elsewhere (Woodman, 1980; Sato and Woodman, 1982a,b). In Section 3, we estimate the vertical scale and dominant period of the observed quasi-periodic oscillation. Then, in Section 4 the vertical propagation characteristics of the wave are investigated, taking into consideration the Doppler shift effect due to the mean zonal flow. In particular, the upward passage of the observed wave packets, which depends on vertical group velocities, is demonstrated; this makes the best use of the fine resolution radar observations. Some quantitative results concerned with the nature of the waves are then obtained, based on the dispersion equation for internal gravity waves with the Coriolis frequency $f=2 \Omega_{z}$ included ( $f$-plane analysis). Finally, in Section 5 another type of oscillation with somewhat larger vertical scale is presented, and its relationship with the inertia-gravity waves is discussed.

\section{The experiment}

The observational periods of the radar experiments were 25-26 June 1979, 10-30 October 1979 and 2022 March 1981. Zonal and meridional winds were alternately observed at intervals of about one hour by steering the antenna beam eastward and northward at $7.5-15^{\circ}$ zenith angle. These observations achieved a time resolution of $1-2 \mathrm{~min}$ and an height resolution of $150 \mathrm{~m}$, by using a complementary code scheme as reported by Woodman (1980). The observed height range was $5-30 \mathrm{~km}$. The radar echoes were processed in real time using an array processor and stored on magnetic tape in the form of power spectra. The wind velocity was deduced from the resulting power spectra using the analysis program of Sato and Woodman (1982b). The observed line-of-sight velocity was averaged over about one hour while the antenna beam was directed toward the same position, giving the hourly zonal and meridional wind values. This procedure serves to avoid contamination of vertical wind components and to increase the statistical reli- ability of the deduced data, since the vertical velocity associated with atmospheric motions with periods longer than one hour is much smaller than the corresponding horizontal velocity.

\section{Time-mean and fluctuating winds}

Figure 1 shows vertical profiles of the time-mean zonal winds (left) and time-height sections of the fluctuating wind component around the mean value (right) observed in October 1979 (top) and March 1981 (bottom). Wind direction is defined positive westerly. The arrows indicate the tropopause level deduced from rawinsonde temperature data at San Juan $(80 \mathrm{~km}$ east of Arecibo). In the left-hand diagrams, the thin lines each side of the thick line indicate the standard deviation from the mean value. During the October 1979 observation period, the mean zonal winds have fairly small values in the upper troposphere, while the easterly winds increase with increasing height in the lower stratosphere, reaching $-20 \mathrm{~m} \mathrm{~s}^{-1}$ around $22 \mathrm{~km}$. During the March 1981 observation period, although westerly winds of $15-20 \mathrm{~m} \mathrm{~s}^{-1}$ are dominant in the troposphere, the wind direction turns easterly at $21-22 \mathrm{~km}$. Thus, the vertical wind shear is easterly with increasing height in the lower stratospheric region during both of these periods.

The fluctuating components are illustrated in Fig. 1 by contour plots at $2 \mathrm{~m} \mathrm{~s}^{-1}$ intervals; shaded areas denote easterly winds. It can be seen that the characteristics of the wind variations are considerably different between the troposphere and the stratosphere. In the lower stratosphere, a thin, multilayered wind structure is generally prominent, and the vertical scale of the fluctuation is particularly small below 20 $\mathrm{km}$. In the upper troposphere, on the other hand, the wind structure shows a larger vertical scale. During the October 1979 period, in particular, the wind direction varies almost in phase within the height range $10-16 \mathrm{~km}$.

In order to clarify the small vertical-scale fluctuation, a high-pass filtering is applied to the vertical wind profile at each observation time; the cutoff length of the filter is $5 \mathrm{~km}$. Figure 2 shows the results in a form of contour plots similar to Fig. 1. Here, rms amplitudes of the fluctuations at each height are presented on the right-hand side. There appears to be a distinct quasi-periodic $2-3 \mathrm{~m} \mathrm{~s}^{-1} \mathrm{rms}$ amplitude oscillation in the $16-20 \mathrm{~km}$ height range. A characteristic time scale of the oscillation is estimated to be $\sim$ 1-2 days, being much longer in March 1981 than in October 1979. The phase of the oscillation shows a marked downward propagation in time, indicating atmospheric waves which transport energy upward. These features are also detected in the meridional wind fluctuations during each observation.

Inspection of the fluctuation in more detail, how- 
$10-13$ OCT 1979
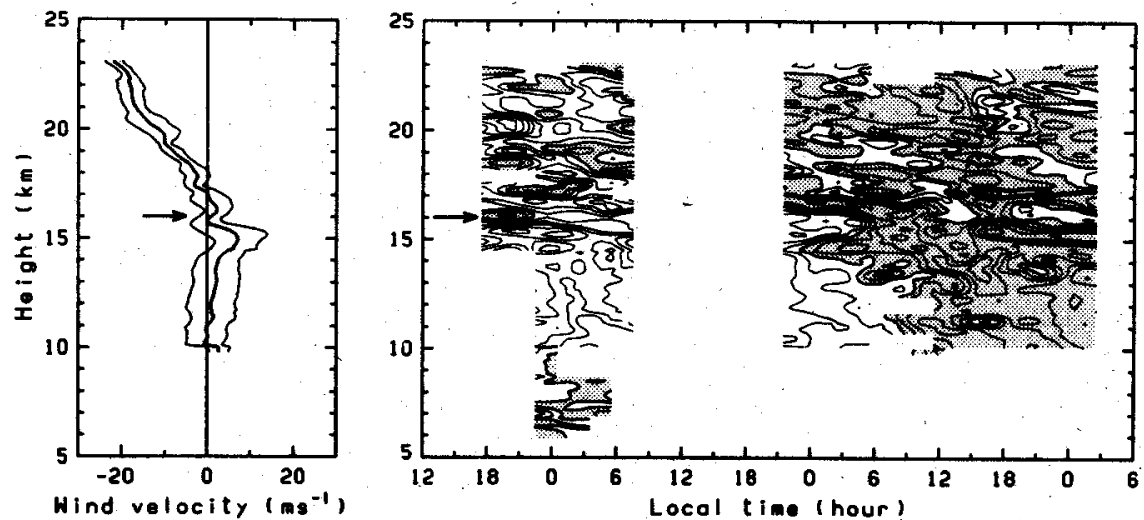

20 - 22 MAR 1981
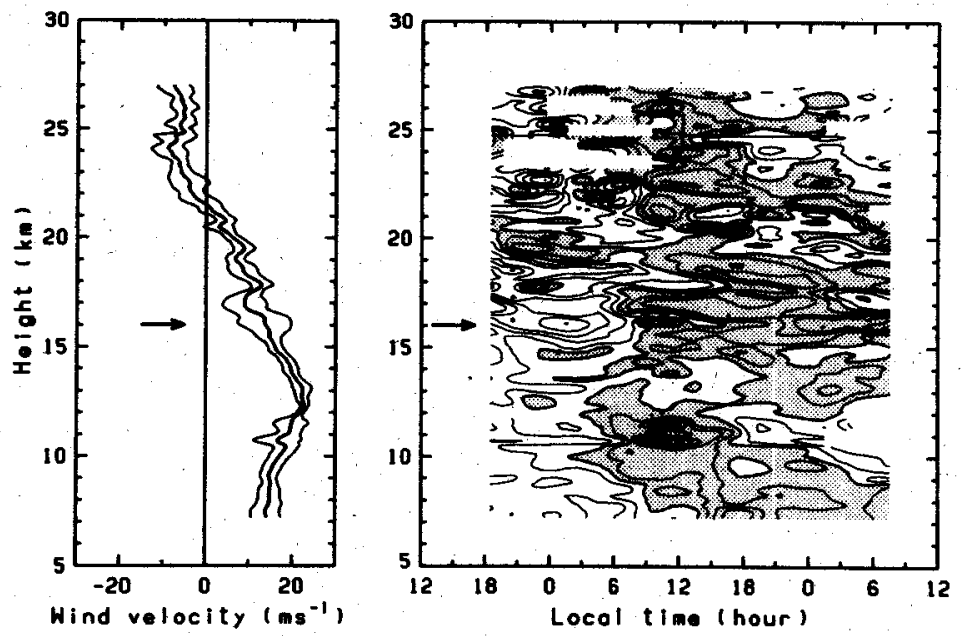

FIG. 1. Vertical profiles of the time-mean zonal winds (left), and time-height sections of the fluctuating wind components around the mean value (right) observed in October 1979 (top) and March 1981 (bottom). The wind direction is defined positive westerly, and the shaded areas denote negative (easterly) winds. The two thin lines each side of the thick line (left) show the standard deviation from the mean. Arrows indicate the tropopause level and contours are at $2 \mathrm{~m} \mathrm{~s}^{-1}$ intervals.

ever, indicates that the quasi-periodic oscillation is not so clearly seen above $20 \mathrm{~km}$, where a discontinuity in the phase propagation is found. This feature implies that the characteristic of the fluctuations changes at this level. To confirm this, we make a cross-correlation analysis of the small vertical-scale wind fluctuation between each height level and a specified reference height level. Figure 3 illustrates the results for the correlation larger than 0.5 in the form of contour plots with a 0.2 interval. The $18 \mathrm{~km}$ height level (dashed line) where the oscillation of interest is most obviously observed is taken as the reference level. The cross correlation is actually calculated within the 13-15 h lag time for statistical reliability. It is evident from the dominance of the cross correlation that the phase of the oscillation continuously propagates within the height range $16-20 \mathrm{~km}$. Thus, this wave seems to appear at the tropopause level, propagate upward, and disappear around $20 \mathrm{~km}$. The vertical wavelength is, on average, inferred to be $\sim 2 \mathrm{~km}$ in both October 1979 and March 1981. Moreover, the vertical phase speed seems to become gradually slower with increasing height. This feature suggests that the wave interacts with the easterly-sheared mean zonal wind (see the next section).

In order to determine the mode of the observed wave, we will estimate the dominant wave period more precisely. In these observations, however, the data length is so short compared with the time scale of the wave that a normal spectral analysis is difficult 
$10-13$ OCT 1979
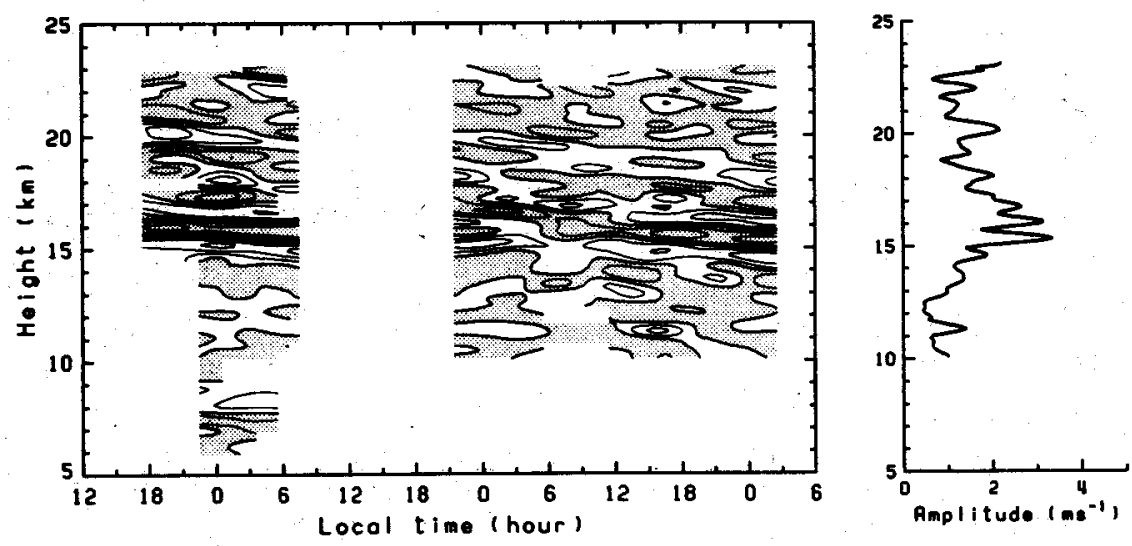

20 - 22 MAR 1981
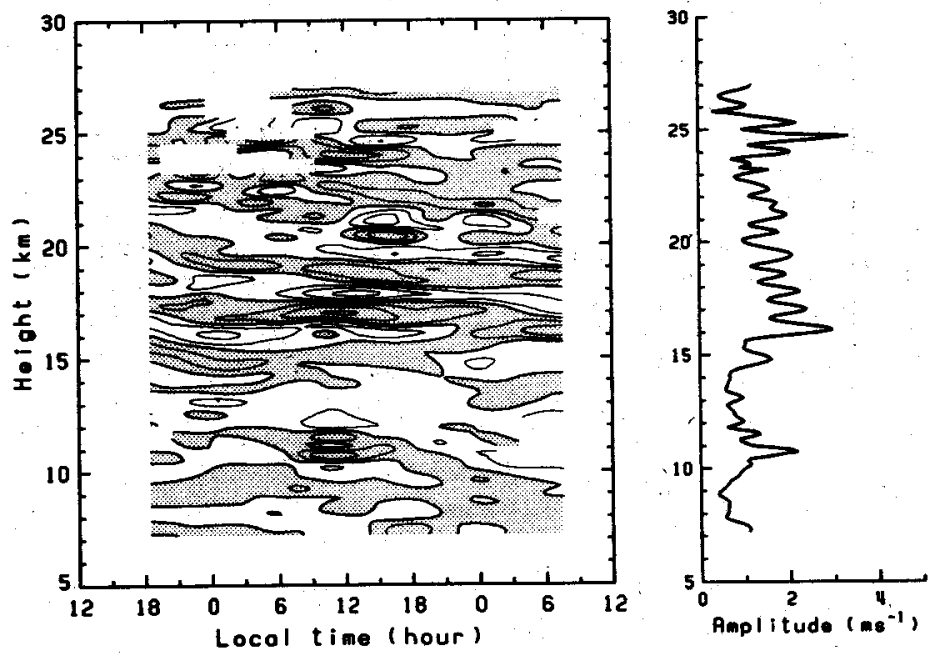

FIG. 2. Time-height sections of the fluctuating wind component with vertical scale shorter than $5 \mathrm{~km}$ (left), and vertical profiles of the rms amplitude of the fluctuations (right). Wind direction and contour intervals as in Fig. 1.
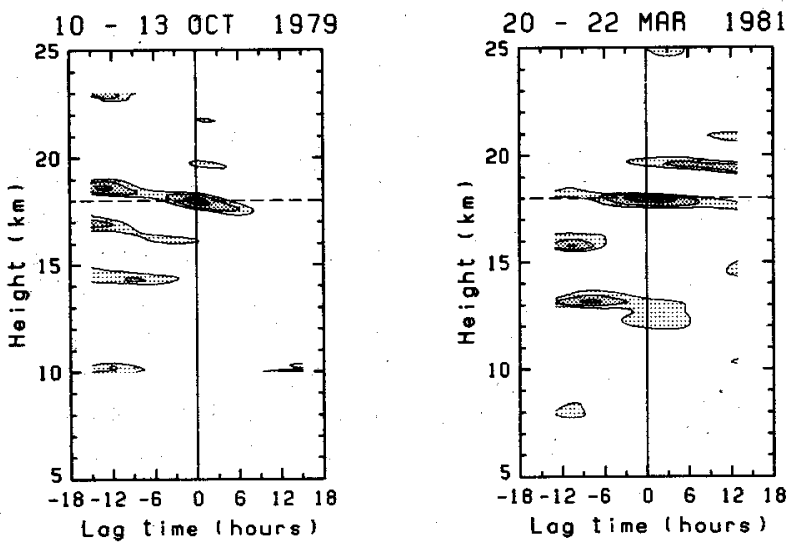

FIG. 3. Cross correlations between each height level and a specified reference height level. Contour lines are at 0.2 intervals for correlations larger than 0.5 , and dashed lines show the reference height level. to perform: As an alternative, sinusoidal functions with periods from 10 to $100 \mathrm{~h}$ are successively fitted to both the zonal and meridional components in a least-mean-square sense in the height range 17-19 $\mathrm{km}$ where the oscillation is especially prominent. For each period, we examine the ratio of the fitting residual to the resulting amplitude, and average it over 14 levels in the height range considered. Figure 4 shows the averaged ratio plotted against the period. Defining the period which minimizes the ratio as the predominant period $T_{0}$, we get values of $23 \mathrm{~h}$ for October 1979 and $54 \mathrm{~h}$ for March 1981, as shown by the arrows.

In this harmonic fitting procedure we assume a long duration for the quasi-periodic oscillation, but in view of statistical reliability this could be very misleading. However, the long-period oscillation has been detected in every observational case including 

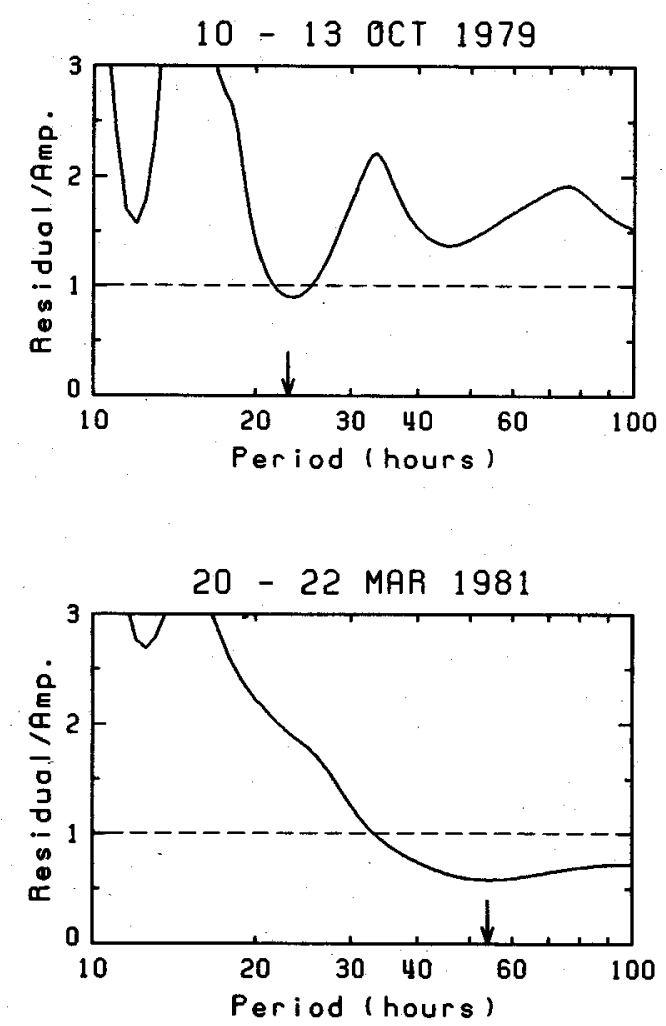

FIG. 4. Ratios of residuals (standard deviation) to amplitudes derived from a least-mean-square fitting of sinusoidal functions with various periods $(10-100 \mathrm{~h}$ ) to both zonal and meridional wind components in the height range $17-19 \mathrm{~km}$. The resulting dominant periods are shown by the arrows.

that of 25-26 June 1979 and a number of other fragmentary observations over Arecibo during 197981. Furthermore, Figs. 2 and 3 show that at least two or three cycles of the oscillation can be traced in the vertical direction. Therefore, it is considered that the oscillation exists for a sufficiently long period in this height range.

The observed periods of 23 or $54 \mathrm{~h}$ are too short for an equatorial planetary-scale wave such as a mixed Rossby-gravity wave. This range of periods and the very short vertical scale derived above suggest that the oscillation is an internal inertia-gravity wave. In the equatorial region, gravity-type waves with periods near to or longer than a day can propagate into the stratosphere, since the Coriolis frequency $f$ $=2 \Omega_{z}$ (i.e., the lower cutoff frequency of internal gravity waves) becomes small. In the present case, this value is $38.2 \mathrm{~h}$ in terms of the cutoff period (inertial period $T_{c}=2 \pi / f$ ) at the latitude of Arecibo $\left(18.4^{\circ} \mathrm{N}\right)$. The period observed in March 1981 is, however, somewhat longer than this cutoff period, although the vertical propagation of the wave is clearly detected. This inconsistency is possibly explained by a Doppler shift effect due to the westerly mean zonal wind, as will be discussed later.

\section{Smaller vertical-scale oscillations}

In order to better understand the vertical propagation of the observed wave, it is first necessary to determine the intrinsic frequency $\omega$ :

$$
\omega=\sigma-k_{x} U_{0},
$$

where $\sigma=2 \pi / T_{0}$ is the observed (apparent) frequency, $k_{x}$ the horizontal wavenumber, and $U_{0}$ the mean zonal flow. Equation (1) shows that $\omega$ is a function of $U_{0}$ at each height. Inherently, both the horizontal scale (or $k_{x}$ ) and propagation direction of atmospheric waves cannot be determined unless they are observed simultaneously at a number of stations. In the present case, however, we can fortunately determine the height levels where the wave appears and disappears, as shown in Fig. 3. This information can be used to infer the horizontal phase speed, by interpreting the disappearance of the wave as a critical-level absorption by the easterly-sheared mean flow:

It should be noted that the conditions for such a wave-flow interaction are different according to a horizontal scale of the waves. For planetary-scale waves (Lindzen, 1970), absorption at a critical level occurs when the horizontal phase speed $c$ equals the mean wind $U_{0}$. Supposing the October 1979 case to be a planetary-scale wave, the phase speed of about $-10 \mathrm{~m} \mathrm{~s}^{-1}$. (westward) is inferred from the mean zonal wind $U_{02}$ around the height $z_{2}$ where the wave disappears. During this observation, the mean wind velocity $U_{01}$ at the tropopause level $z_{1}$ where the wave appears is so small that we may approximately take the observed period $T_{0}=23 \mathrm{~h}$ and the phase speed to be relative to the flow at this height. Thus the horizontal wavelength is given as

$$
\lambda_{x}=|c| T_{0} \approx 1000 \mathrm{~km} \text {. }
$$

Another possible reason for the disappearance of the planetary-scale waves is that they are confined closer to the equator as the intrinsic frequency $\omega$ approaches the Coriolis frequency $f$. From the condition $\omega=f$, the horizontal wavenumber is given, using Eq. (1), by

$$
k_{x}=\frac{\sigma-f}{U_{02}}
$$

This relationship gives a horizontal wavelength $\lambda_{x}$ $=2 \pi / k_{x}$ of $\sim 2000 \mathrm{~km}$. In view of the deduced horizontal scales of $1000-2000 \mathrm{~km}$, it is permissible to use the $f$-plane analysis, and to consider the observed waves to be a mesoscale or cloud-clusterscale feature.

In the case of internal inertia-gravity waves on the $f$-plane, the wave absorption due to the flow takes place at the height where $\omega$ reaches $f$ (Booker and Bretherton, 1967). We can infer the horizontal wavenumber from the same relationship as Eq. (3). Table 1 summarizes the resulting intrinsic periods $T_{1}=2 \pi / \omega_{1}$ and 
TABLE 1. Characteristics of the smaller vertical-scale wave.

\begin{tabular}{|c|c|c|c|c|}
\hline : & & $\begin{array}{l}\text { June } \\
1979\end{array}$ & $\begin{array}{c}\text { October } \\
1979\end{array}$ & $\begin{array}{c}\text { March } \\
1981\end{array}$ \\
\hline $\begin{array}{l}U_{01} \\
U_{02} \\
z_{1} \\
z_{2} \\
T_{0} \\
T_{1} \\
V_{p x} \\
\lambda_{x} \\
\lambda_{21} \\
\lambda_{2}\end{array}$ & $\begin{array}{l}\text { Mean zonal wind at } z_{1}\left(\mathrm{~m} \mathrm{~s}^{-1}\right) \\
\text { Mean zonal wind at } z_{2}\left(\mathrm{~m} \mathrm{~s}^{-1}\right) \\
\text { Tropopause height }\left(\mathrm{km}^{-1}\right. \\
\text { Wave-absorbed height }(\mathrm{km}) \\
\text { Observed period (h) } \\
\text { Intrinsic period (h) relative to } \\
\quad \text { mean zonal wind at } z_{1} \\
\left.\text { Horizontal phase velocity ( } \mathrm{m} \mathrm{s}^{-1}\right) \\
\quad \text { relative to mean zonal wind at } \\
\quad z_{1} \\
\text { Horizontal wavelength }(\mathrm{km}) \\
\text { Vertical wavelength at } z_{1}(\mathrm{~km}) \\
\text { Vertical wavelength }(\mathrm{km}) \text { at the } \\
\text { height of } 18 \mathrm{~km}\end{array}$ & $\begin{array}{c}-4.3 \\
-14 \\
17 \\
20 \\
18 \\
\\
21\end{array}$ & $\begin{array}{c}2.6 \\
-8.0 \\
16 \\
19 \\
23 \\
20\end{array}$ & $\begin{array}{l}13 \\
5.7 \\
16 \\
20 \\
54 \\
\\
28\end{array}$ \\
\hline
\end{tabular}

horizontal phase velocities relative to the mean flow $V_{p x}=\omega_{1} / k_{x}$ at the tropopause level $z_{1}$, together with the horizontal wavelength $\lambda_{x}=2 \pi / k_{x}$. The observed wave period $T_{0}$, and the background mean zonal winds $U_{01}$ and $U_{02}$ at the heights $z_{1}$ and $z_{2}$, respectively, are also given. In this calculation, we determine the wave-absorbed height $z_{2}$ at $19 \mathrm{~km}$ for October 1979 and $20 \mathrm{~km}$ for March 1981 in light of the crosscorrelation analysis shown in Fig. 3. In Table 1, results deduced from the June 1979 observation are also cited, since similar inertia-gravity wave characteristics are obtained. It is remarkable that the intrinsic period at $z_{1}$ lies in the range 20-30 h in every observation, although the apparent periods observed on the ground differ greatly depending on the individual mean flow. The other parameters such as horizontal phase velocity and wavelength are also approximately equal among the three observations, indicating the similarity of these observed waves. Furthermore, these characteristics are in good agreement with those of the westward-propagating waves reported by Cadet and Teitelbaum (1979). It is particularly noteworthy that the possibility of vertical propagation as the internal mode is demonstrated for the wave observed in March 1981, whose period is apparently longer than the cutoff period $T_{c}$. In this case, the observed period can be interpreted to be Doppler-shifted to the longer-period side by the strong westerly mean zonal wind at the tropopause level, which is in the opposite direction to the horizontal phase speed.

The vertical wavelength $\lambda_{z}=2 \pi / k_{z},\left(k_{z}\right.$ is the vertical wavenumber) is inferred from the dispersion equation on the $f$-plane (e.g., Gossard and Hooke, 1975):

$$
k_{z}{ }^{2}=\frac{N^{2}}{\omega^{2}-f^{2}} k_{x}^{2},
$$

where $N$ is the Brunt-Väisälä frequency which is estimated from the San Juan balloon temperature data. The deduced vertical wavelength at the tropopause level is $5-7 \mathrm{~km}$ in every observational case (Table 1). It shortens to $2-3 \mathrm{~km}$ at $18 \mathrm{~km}$, being in good agreement with the observed result presented in the previous section. It is difficult, however, to deduce the vertical phase velocity exactly from the observation because of the limited observation period, and so comparison is not easily made between theory and observation at each height level.

Further information on wave propagation can be obtained from a modulation pattern of the observed oscillation, i.e., a passage of wave packets, taking advantage of the high resolution of the present observations. Figure 5 shows the detailed structure of both zonal and meridional wind fluctuations observed on 11-13 October 1979 in the height range $15-20 \mathrm{~km}$. Contour lines are drawn at $1 \mathrm{~m} \mathrm{~s}^{-1}$ intervals in order to magnify the oscillation. Shaded areas denote regions of easterly and northerly winds for the zonal and meridional components, respectively. Dashed lines represent the traces of wave packets; they simply connect local maxima of the positive and negative wind velocities shown by crosses. It is remarkable that these lines for both zonal and meridional components pass through almost the same time-height locations. In addition, the directions of wind velocity vectors along each line rotate clockwise when traced upward with time, being consistent with the internal gravity-wave mode in the Northern Hemisphere. Such systematic tracing is possible up to the $18.5 \mathrm{~km}$ level.

The theoretical vertical group velocity $V_{g z}=\partial \omega /$ $\partial k_{\mathrm{z}}$ is given by

$$
V_{g z}=-\left(\omega^{2}-f^{2}\right) / k_{z} \omega
$$

The gradients of the arrows on the left side of Fig. 5 show the vertical group velocities deduced from Eq. (5). They agree fairly well with the gradients of the dashed lines at each height. Therefore, it is concluded that the wave packets which transport wave energy are propagating upward with speed $V_{g z}$. As the wave packets approach the critical level, the upward propagation gradually becomes slower, possibly due to the decrease in $\omega$ through the Doppler-shift effect.

\section{Larger vertical-scale oscillations}

In the previous section we discussed the wind oscillation with a shorter vertical wavelength. As shown in Fig. 1, however, another type of wind fluctuation with somewhat longer vertical wavelength can be seen in the height range above $20 \mathrm{~km}$, where the smaller vertical-scale wave is considered not to exist. In this section, we will study the nature of this type of fluctuation.

Wind fluctuations with vertical scales larger than $5 \mathrm{~km}$ are extracted with the aid of low-pass filtering, contrary to the method of Section 3 . This procedure excludes the smaller vertical-scale wave discussed earlier. Figure 6 shows, in a similar manner to Fig. 
ZONAL WIND

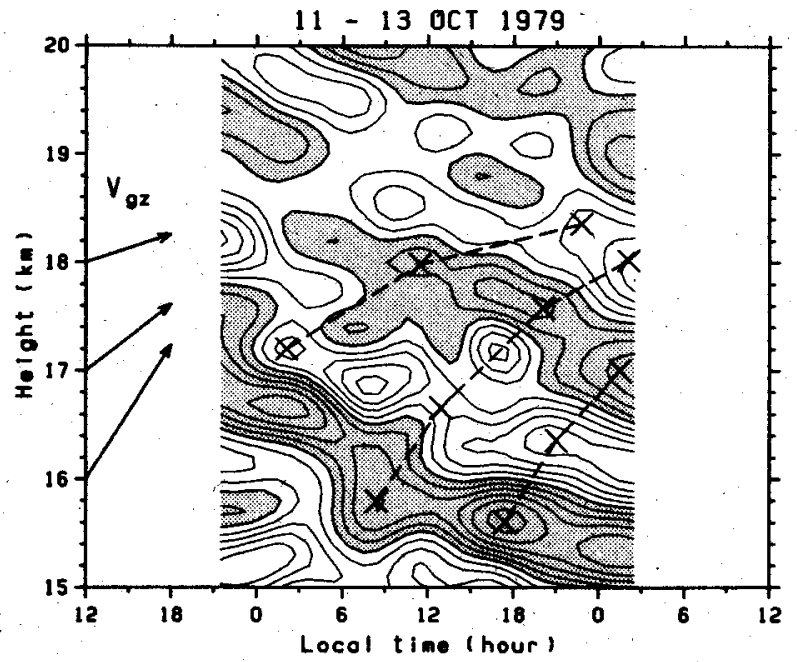

MERIDIONAL WIIND

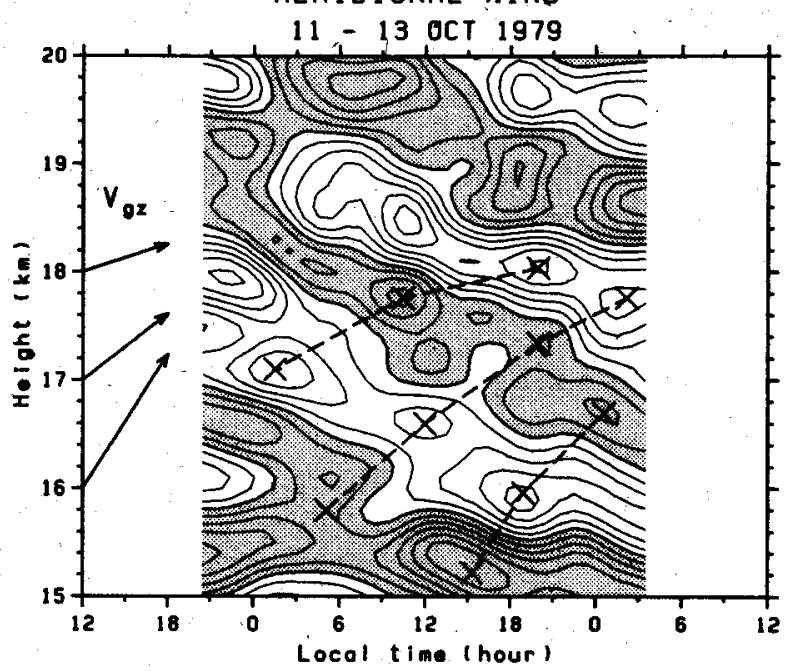

FIG. 5. Detailed time-height sections of wind fluctuations in the height range $15-20 \mathrm{~km}$ for zonal and meridional components. Contours are at $1 \mathrm{~m} \mathrm{~s}^{-1}$ intervals. Shaded areas denote easterly or northerly winds, and dashed lines show the passage of the observed wave packets, while the gradients of the arrows denote the vertical group velocities inferred from Eq. (5).
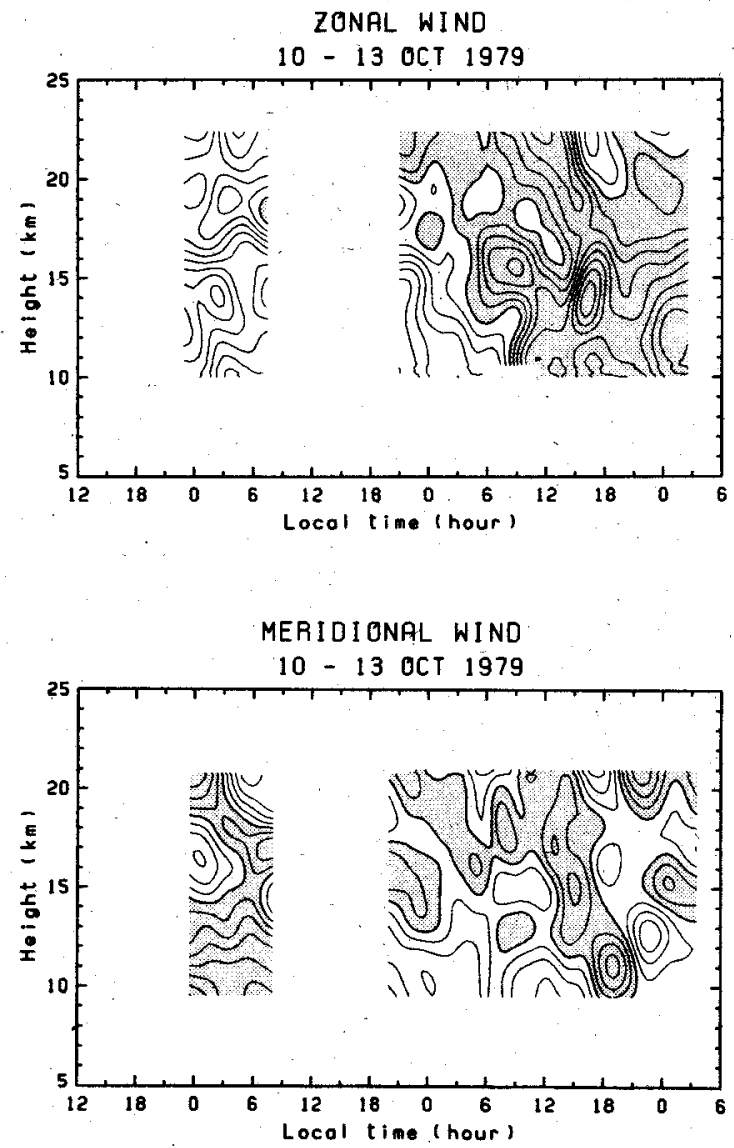

FIG. 6. Time-height sections of wind fluctuations with vertical scale larger than $5 \mathrm{~km}$ for the zonal (top) and meridional (bottom) wind components observed in October 1979. Contours are at $1 \mathrm{~m}$ $\mathrm{s}^{-1}$ intervals. Shaded areas denote easterly or northerly winds.
2 , the resulting time-height sections for the zonal and meridional wind components observed in October 1979. This figure reveals the dominance of a quasiperiodic oscillation above the tropopause height (16 $\mathrm{km}$ ), which shows a rather fast descending phase in time. This phase propagation is more obvious with the meridional component, since the meridional wind has almost no trend over the observed period. The dominant period $T_{0}^{*}$ estimated in the same manner as in Section 3 is $16-17 \mathrm{~h}$ in both the October 1979 and March 1981 cases.

The amplitude and phase of the oscillations are then deduced by a least-mean-square fitting with period $T_{0}^{*}$ at each height, since the data length covers at least two or three cycles of the oscillation in this case. Figure 7 shows the height profiles of the amplitude and phase in the height range $15-25 \mathrm{~km}$. Thick and thin lines indicate the zonal and meridional components, respectively. The phase is represented by the local time when the wind velocity becomes maximum westerly (southerly) for the zonal (meridional) component on 12 October 1979 and 21 March 1981 in the respective observational periods. The scale of the meridional phase is shifted by $T_{\delta}^{*} / 4\left(90^{\circ}\right)$, in order to clarify the phase quadrature relationship. The amplitude is $1-2 \mathrm{~m} \mathrm{~s}^{-1}$, and the phase shows a downward propagation above the tropopause level. It is apparent that this oscillation has the nearly circular polarization and the clockwise rotation of the wind velocity vector. These characteristics of the periods and the phase quadrature relationship also conform to those of the internal inertia-gravity waves in the Northern Hemisphere. It is of particular interest that the vertical phase velocity (gradient of the plotted 
$10-13$ OCT 1979
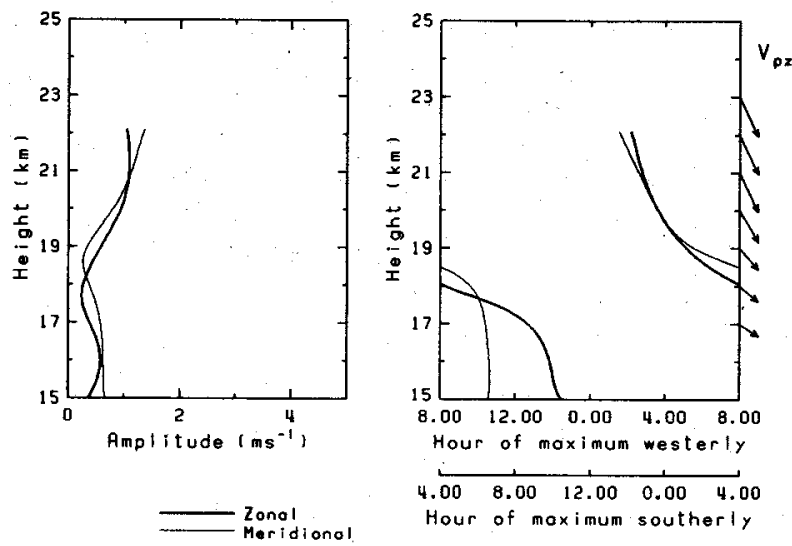

$\begin{array}{llll}4.00 \quad 8.00 & 12.00 & 0.00 & 4.00\end{array}$ Hour of maximum southerly

$20-22$ MAR 1981
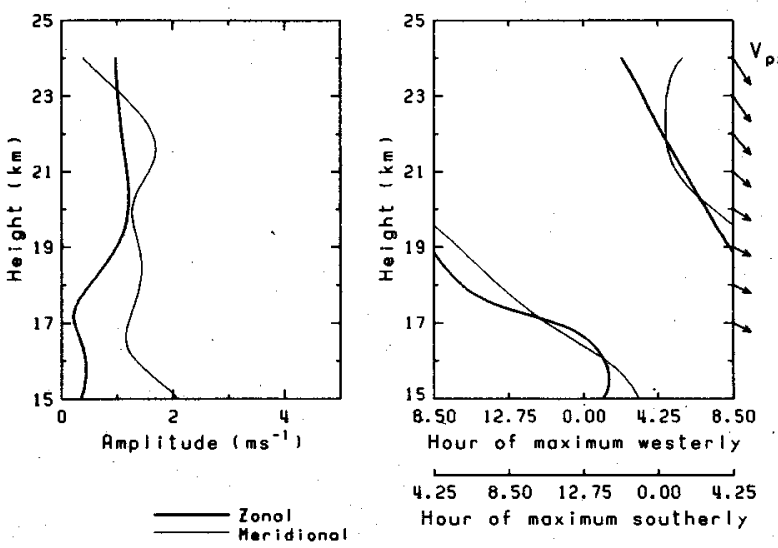

FIG. 7. Amplitude and phase of the larger vertical-scale oscillations estimated by a least-mean-square fitting in the lower stratosphere. Thick and thin lines indicate the zonal and meridional components, respectively. The hour of the phase is LST on 12 October 1979 or 21 March 1981. The scale of the meridional phase is shifted by $T_{\delta}^{*} / 4\left(90^{\circ}\right)$ in order to clarify the phase quadrature relationship. The gradients of the arrows show the vertical phase velocitie deduced from the dispersion equation.

curves) increases with height. This is interpreted as an increase of intrinsic frequency $\omega^{*}$ which is due to a Doppler shift by the easterly-sheared mean zonal wind, contrary to the smaller vertical-scale wave mentioned earlier.

These internal inertia-gravity waves are regarded as a kind of forced oscillation, and so the horizontal scale of the waves depends upon the distribution of the energy source for excitation below the tropopause level. If we assume that both waves are excited by the same energy source, these waves are considered to have similar horizontal scale and intrinsic frequency at tropopause height. In this case, the difference in the apparent vertical scale between the two waves may be ascribed to their horizontal propagation direction. Based on this consideration, we tentatively assume horizontal wavenumber $k_{x}^{*}$ and intrinsic fre- quency $\omega_{1}^{*}$ for the larger vertical-scale oscillation at the tropopause level:

$$
k_{x}^{*}=k_{x}, \quad \omega_{1}^{*}=-\omega_{1},
$$

where $k_{x}$ and $\omega_{1}$ are for the smaller vertical-scale oscillation discussed in the previous section. Then, the apparent frequency relative to the ground is expressed by

$$
\sigma^{*}=\omega_{1}^{*}+k_{x}^{*} U_{01}=-\omega_{1}+k_{x} U_{01} .
$$

The period inferred from this equation closely agrees with the value observed in each experiment (see Table 2 ). This result strongly supports our assumption about the wave parameters $k_{x}^{*}$ and $\omega_{1}^{*}$.

The vertical phase velocity $V_{p z}^{*}=\omega^{*} / k_{z}^{*}$ is calculated using the dispersion equation (4). In Fig. 7, the deduced phase propagation per hour is shown by the arrows on the right. It is apparent that the inferred and observed values are generally in good agreement. This result also verifies the close relationship between the smaller and larger vertical-scale waves.

\section{Concluding remarks}

Tropical wave motion with time scales of 1-2 days has been studied using lower stratospheric wind data obtained by the high-power UHF radar at Arecibo, Puerto Rico $\left(18.4^{\circ} \mathrm{N}, 66.8^{\circ} \mathrm{W}\right)$. The high resolution, especially in height, of these observations made it possible to demonstrate clearly the downward phase propagation and associated upward passage of the wave packets. The wave motion shows significant differences between vertical scales smaller and larger than $5 \mathrm{~km}$. The smaller vertical-scale wave is shown to disappear via a wave-flow interaction at a critical level of $\sim 20 \mathrm{~km}$ where the mean zonal wind has easterly shear with height. These internal inertiagravity waves correspond to mesoscale or cloudcluster-scale wind disturbances, in view of the deduced horizontal scale of $\sim 2000 \mathrm{~km}$.

The relationship between the two wave modes was discussed, taking into consideration the characteristics of their vertical propagation in the shear flow. In order to explain the differences in their vertical propagation characteristics, we assumed westward and eastward propagation for the smaller and larger ver-

TABLE 2. Period of the larger vertical-scale wave: comparison between the wave derived from the observed wind oscillation and that inferred with the same intrinsic period as the smaller verticalscale wave.

\begin{tabular}{lccc}
\hline \hline & $\begin{array}{c}\text { June } \\
1979\end{array}$ & $\begin{array}{c}\text { October } \\
1979\end{array}$ & $\begin{array}{c}\text { March } \\
1981\end{array}$ \\
\hline$T_{\delta}^{*}$ Observed period (h) & 24 & 16 & 17 \\
$T^{*}$ Inferred period from Eq. (6) (h) & 26 & 18 & 19 \\
\hline
\end{tabular}


tical-scale waves, respectively. At the tropopause level, we assumed the same intrinsic period and horizontal scale for the eastward- and westward-propagating waves, based on the disappearance of the latter at a critical level. These assumptions were verified by comparing the deduced vertical phase and group velocity with the corresponding observed values at each height.

It is interesting that these waves seem to be excited as standing waves below the tropopause level. The generation of this type of inertia-gravity wave has been ascribed to the instability of the tropospheric jet stream (Thompson, 1978; Cadet and Teitelbaum, 1979). In the present case over the Caribbean region, however, the activity of cumulus convection may also be related to wave generation, as is the case for wave disturbances in the tropical western Pacific (Wallace, 1971).

There will, of course, be a need for further observations to investigate the nature of wave propagation and excitation in more detail. It will be particularly important to observe such inertia-gravity waves at other locations in different topography. Therefore, we strongly propose that radar wind observations with fine height resolution should be performed soon using the Jicamarca, Peru $\left(11.95^{\circ} \mathrm{S}, 76.87^{\circ} \mathrm{W}\right)$ VHF radar, which is the only high-power radar in the equatorial region other than Arecibo.

Acknowledgments. The authors wish to thank Drs. I. Hirota and K. Fukuyama of Kyoto University for their important advice and constructive comments. Thanks are due also to Dr. T. Matsuno of the University of Tokyo, whose suggestions have significantly contributed to this work. Deep appreciation is due to the reviewers, especially Prof. J. R. Holton, for valuable suggestions on revising the manuscript. One of the authors (SF) is also grateful to Dr. H. Teitelbaum of Service d'Aéronomie du CNRS, France, for his helpful discussion. The Arecibo Observatory is operated by Cornell University under contract with the U.S. National Science Foundation. This work was supported partially by the Nissan Science Foundation of Japan and partially by the National Aero- nautics and Space Administration under NASA Order No. W-14 569.

\section{REFERENCES}

Balsley, B. B., and K. S. Gage, 1980: The MST radar technique: Potential for middle atmosphere studies: Pure Appl. Geophys., $118,452-493$.

Booker, J. R., and F. P. Bretherton, 1967: The critical layer for internal gravity waves in a shear flow. J. Fluid. Mech., 27, 513-539.

Cadet, D., and H. Teitelbaum, 1979: Observational evidence of internal inertia-gravity waves in the tropical stratosphere. $J$. Atmos. Sci., 36, 892-907.

Gossard, E. E., and W. H. Hooke, 1975: Waves in the Atmosphere. Elsevier, $456 \mathrm{pp}$.

Holton, J. R., and R. S. Lindzen, 1968: A note on "Kelvin" waves in the atmosphere. Mon. Wea. Rev., 96, 385-386.

Lindzen, R. S., 1967: Planetary waves on the beta-plane. Mon. Wea. Rev., 95, 441-451.

- 1970: Internal equatorial planetary-scale waves in shear flow. J. Atmos. Sci., 27, 394-407.

- and T. Matsuno, 1968: On the nature of large scale wave disturbances in the equatorial lower stratosphere. $J$. Meteor. Soc. Japan., 46, 215-221.

Madden, R. A., and E. J. Zipser, 1970: Multi-layered structure of the wind over the equatorial Pacific during the Line Islands experiments. J. Atmos. Sci., 27, 336-343.

Röttger, J., 1980: Structure and dynamics of the stratosphere and mesosphere revealed by VHF radar investigations. Pure Appl. Geophys., 118, 494-527.

Sato, T., and R. F. Woodman, 1982a: Fine altitude resolution radar observations of upper-tropospheric and lower-stratospheric winds and waves. J. Atmos. Sci., 39, 2539-2545.

, and - 1982b: Spectral parameter estimation of CAT radar echoes in the presence of fading clutter. Radio Sci., 17, 817-826.

Thompson, R. O. R. Y., 1978: Observations of inertial waves in the stratosphere. Quart. J. Roy. Meteor. Soc., 104, 691-698.

Wallace, J. M., 1971: Spectral studies of tropospheric wave disturbances in the tropical western Pacific. Rev. Geophys. Space Phys., 9, 557-612.

- , and V. E. Kousky, 1968: Observational evidence of Kelvin waves in the tropical stratosphere. J. Atmos. Sci., 25, 900 907.

Woodman, R. F., 1980: High-altitude-resolution stratospheric measurements with the Arecibo $430-\mathrm{MHz}$ radar. Radio Sci., 15, 417-422.

Yanai, M., and T. Maruyama, 1966: Stratospheric wave disturbances propagating over the equatorial Pacific. J. Meteor. Soc. Japan, 44, 291-294. 